\title{
On the distribution of multivariate Jacobi sums
}

\author{
Qing Lu* Weizhe Zheng ${ }^{\dagger}$
}

\begin{abstract}
Let $\mathbf{F}_{q}$ be a finite field of $q$ elements. We show that the normalized Jacobi sum $q^{-(m-1) / 2} J\left(\chi_{1}, \ldots, \chi_{m}\right)\left(\chi_{1} \cdots \chi_{m}\right.$ nontrivial $)$ is asymptotically equidistributed on the unit circle, when $\chi_{1} \in \mathcal{A}_{1}, \ldots, \chi_{m} \in \mathcal{A}_{m}$ run through arbitrary sets of nontrivial multiplicative characters of $\mathbf{F}_{q}^{\times}$, if $\# \mathcal{A}_{1} \geq q^{\frac{1}{2}+\epsilon}$, $\# \mathcal{A}_{2} \geq(\log q)^{\frac{1}{\delta}-1}$ for $\epsilon>\delta>0$ fixed and $q \rightarrow \infty$ or if $\# \mathcal{A}_{1} \# \mathcal{A}_{2} / q \rightarrow \infty$. This extends previous results of Xi, Z. Zheng, and the authors.
\end{abstract}

Let $\mathbf{F}_{q}$ be a finite field of $q$ elements and let $\mathbf{C}$ be the field of complex numbers. We let $\mathcal{X}_{q}$ denote the set of nontrivial multiplicative characters $\mathbf{F}_{q}^{\times} \rightarrow \mathbf{C}^{\times}$. For $m \geq 2, \chi_{1}, \ldots, \chi_{m} \in \mathcal{X}_{q}$ satisfying $\chi_{1} \cdots \chi_{m} \neq 1$, we consider the Jacobi sum

$$
J\left(\chi_{1}, \ldots, \chi_{m}\right)=\sum_{\substack{a_{1}, \ldots, a_{m} \in \mathbf{F}_{q}^{\times} \\ a_{1}+\cdots+a_{m}=1}} \chi_{1}\left(a_{1}\right) \cdots \chi_{m}\left(a_{m}\right),
$$

which has absolute value $q^{\frac{m-1}{2}}$. For a nonempty subset $\mathcal{A} \subseteq \mathcal{X}_{q}^{m}$, we define the discrepancy $D(\mathcal{A})$, to be the supremum of

$$
\left|\frac{\#\left\{\left(\chi_{1}, \ldots, \chi_{m}\right) \in \mathcal{A}^{\circ} \mid q^{-\frac{m-1}{2}} J\left(\chi_{1}, \ldots, \chi_{m}\right) \in e^{2 \pi i[a, b]}\right\}}{\# \mathcal{A}^{\circ}}-(b-a)\right|
$$

for all real numbers $a \leq b \leq a+1$, where

$$
\mathcal{A}^{\circ}=\left\{\left(\chi_{1}, \ldots, \chi_{m}\right) \in \mathcal{A} \mid \chi_{1} \cdots \chi_{m} \neq 1\right\}
$$

and $e^{2 \pi i[a, b]}$ denotes the image of the interval $[a, b]$ under the map $x \mapsto e^{2 \pi i x}$. For $\mathcal{A}^{\circ}$ empty we adopt the convention that $D(\mathcal{A})=1$.

The goal of this note is to prove the following equidistribution property.

${ }^{*}$ School of Mathematical Sciences, Beijing Normal University, Beijing 100875, China; email: qlu@bnu.edu.cn. Partially supported by Beijing Natural Science Foundation Grant 1202014; Fundamental Research Funds for Central Universities of China; China Scholarship Council.

$\dagger$ Morningside Center of Mathematics and Hua Loo-Keng Key Laboratory of Mathematics, Academy of Mathematics and Systems Science, Chinese Academy of Sciences, Beijing 100190, China; University of the Chinese Academy of Sciences, Beijing 100049, China; email: wzheng@math.ac.cn. Partially supported by National Natural Science Foundation of China Grants 11822110, 11688101, 11621061. 
Theorem 1. There exists a constant $C$ such that for all $q$ and all integers $m \geq 2$, $s \geq 1$ and for all nonempty subsets $\mathcal{A}_{1} \subseteq \mathcal{X}_{q}, \mathcal{A}_{2} \subseteq \mathcal{X}_{q}, \mathcal{B} \subseteq \mathcal{X}_{q}^{m-2}$, we have

$$
\begin{gathered}
D\left(\mathcal{A}_{1} \times \mathcal{A}_{2} \times \mathcal{B}\right) \leq C\left(s\left(\frac{\sqrt{q}}{A_{1}}\right)^{\frac{1}{2 s+1}}+\frac{1}{\sqrt{s A_{2}}}\left(\frac{q}{A_{1}}\right)^{\frac{1}{2 s}} \log q\right) \\
D\left(\mathcal{A}_{1} \times \mathcal{A}_{2} \times \mathcal{B}\right) \leq C\left(\frac{q}{A_{1} A_{2}}\right)^{\frac{1}{4}}
\end{gathered}
$$

Here $A_{i}=\# \mathcal{A}_{i}$.

Corollary 2. (i) Let $0<\epsilon \leq \frac{1}{2}$ and $c>0$ be real constants. Then for nonempty subsets $\mathcal{A}_{1} \subseteq \mathcal{X}_{q}, \mathcal{A}_{2} \subseteq \mathcal{X}_{q}, \mathcal{B} \subseteq \mathcal{X}_{q}^{m-2}$ satisfying $\# \mathcal{A}_{1} \geq c q^{\frac{1}{2}+\epsilon}$, we have $D\left(\mathcal{A}_{1} \times \mathcal{A}_{2} \times \mathcal{B}\right) \rightarrow 0$ as

$$
\frac{\# \mathcal{A}_{2}}{(\log q)^{\frac{1}{\epsilon}-1}(\log \log q)} \rightarrow \infty
$$

(ii) For nonempty subsets $\mathcal{A}_{1} \subseteq \mathcal{X}_{q}, \mathcal{A}_{2} \subseteq \mathcal{X}_{q}, \mathcal{B} \subseteq \mathcal{X}_{q}^{m-2}, D\left(\mathcal{A}_{1} \times \mathcal{A}_{2} \times \mathcal{B}\right) \rightarrow 0$ as $\# \mathcal{A}_{1} \# \mathcal{A}_{2} / q \rightarrow \infty$.

Remark 3. (i) For $m=2$, the problem of asymptotic equidistribution of

$$
\left\{q^{-1 / 2} J\left(\chi_{1}, \chi_{2}\right)\right\}_{\chi_{1} \in \mathcal{A}_{1}, \chi_{2} \in \mathcal{A}_{2}, \chi_{1} \chi_{2} \neq 1}
$$

was first suggested by Shparlinski [S, Section 5].

(ii) The case $s=1$ of (11) was proved in [LZZ, (1.2)], which implies $D\left(\mathcal{A}_{1} \times\right.$ $\left.\mathcal{A}_{2} \times \mathcal{B}\right) \rightarrow 0$ as $\frac{A_{1} A_{2}}{q \log ^{2} q} \rightarrow \infty$. The case $m=2$ of (11) is a theorem of $\mathrm{Xi}$ $[\mathrm{X}$, Theorem 1.5] and the problem of generalization to multivariate Jacobi sums was suggested by him in $[\mathrm{X}]$.

(iii) The bound (2) improves [LZZ, (1.3)]. Note that (2) is stronger than (11) when $\left(A_{1} q\right)^{1 / 3} \leq A_{2} \leq A_{1}$.

(iv) It follows from a theorem of Katz [K, Theorem 9.5] (see also [LZZ, (1.7)] for the case $m=2$ ) that for fixed $\chi_{2}, \ldots, \chi_{m} \in \mathcal{X}_{q}$ satisfying $\chi_{2} \cdots \chi_{m} \neq \mathbf{1}$,

$$
\left\{q^{-(m-1) / 2} J\left(\chi_{1}, \ldots, \chi_{m}\right)\right\}_{\chi_{1} \in \mathcal{X}_{q}, \chi_{1} \cdots \chi_{m} \neq \mathbf{1}}
$$

is asymptotically equidistributed on the unit circle as $q \rightarrow \infty$.

(v) When one or both of the $\mathcal{A}_{i}$ 's are $\mathcal{X}_{q}$, better bounds than Theorem 1 have been given. See [KZ] by Katz and Z. Zheng (for the case $m=2$ and $\mathcal{A}_{1}=\mathcal{A}_{2}=\mathcal{X}_{q}$ ) and [LZZ, Theorems 1.4, 1.6].

Theorem 1 follows from the following bounds of the moments of Jacobi sums, which we deduce from results of Deligne and Katz. Our proof of (3) is similar to that of Xi [X, Theorem 1.4], using Hölder's inequality. Our proof of (44) using Cauchy's inequality was originally presented using matrices indexed by $\widehat{\mathbf{F}_{q}^{\times}}$. Xi suggested the current presentation, similar to the proof of [dIBSSV, Lemma 3.8]. 
Theorem 4. For all integers $m \geq 2, s \geq 1, n \geq 1$ and for all nonempty subsets $\mathcal{A}_{1} \subseteq \mathcal{X}_{q}, \mathcal{A}_{2} \subseteq \mathcal{X}_{q}, \mathcal{B} \subseteq \mathcal{X}_{q}^{m-2}$, we have

$$
\begin{gathered}
\left|M^{(n)}\right| \leq A_{1}^{1-\frac{1}{2 s}}\left(s ! A_{2}^{s} q+s A_{2}^{2 s}(n \sqrt{q}+1)\right)^{\frac{1}{2 s}} B \\
\left|M^{(n)}\right| \leq n \sqrt{A_{1} A_{2} q} B
\end{gathered}
$$

where $M^{(n)}=\sum_{\left(\chi_{1}, \ldots, \chi_{m}\right) \in \mathcal{A}^{\circ}}\left(q^{-\frac{m-1}{2}} J\left(\chi_{1}, \ldots, \chi_{m}\right)\right)^{n}, \mathcal{A}=\mathcal{A}_{1} \times \mathcal{A}_{2} \times \mathcal{B}, A_{i}=\# \mathcal{A}_{i}$, $B=\# \mathcal{B}$.

Proof. We have

$$
M^{(n)}=\sum_{\left(\chi_{3}, \ldots, \chi_{m}\right) \in \mathcal{B}} \sum_{\substack{\chi_{1} \in \mathcal{A}_{1}, \chi_{2} \in \mathcal{A}_{2} \\\left(\chi_{1}, \ldots, \chi_{m}\right) \in \mathcal{A}^{\circ}}}\left(q^{-\frac{m-1}{2}} J\left(\chi_{1}, \ldots, \chi_{m}\right)\right)^{n},
$$

It suffices to prove the stated estimates for the inner sum. Thus we may assume $B=1$. Let $\mathcal{B}=\left\{\left(\chi_{3}, \ldots, \chi_{m}\right)\right\}$ and $\lambda=\chi_{3} \cdots \chi_{m}$. Fix a nontrivial additive character $\psi: \mathbf{F}_{q} \rightarrow \mathbf{C}^{\times}$. For $\chi \in \widehat{\mathbf{F}}_{q}^{\times}=\mathcal{X}_{q} \cup\{\mathbf{1}\}$, consider the Gauss sum

$$
G(\chi)=\sum_{a \in \mathbf{F}_{q}^{\times}} \psi(a) \chi(a)
$$

Recall that $|G(\chi)|=\sqrt{q}$ for $\chi \in \mathcal{X}_{q}$ and $G(\mathbf{1})=-1$. We have

$$
J\left(\chi_{1}, \ldots, \chi_{m}\right)=\frac{G\left(\chi_{1}\right) \cdots G\left(\chi_{m}\right)}{G\left(\chi_{1} \cdots \chi_{m}\right)} .
$$

By the above, we have

$$
\left|M^{(n)}\right| \leq \sum_{\chi_{1} \in \mathcal{A}_{1}}\left|\sum_{\substack{\chi_{2} \in \mathcal{A}_{2} \\ \chi_{1} \chi_{2} \lambda \neq \mathbf{1}}} q^{-n} G\left(\chi_{2}\right)^{n} \overline{G\left(\chi_{1} \chi_{2} \lambda\right)^{n}}\right| .
$$

By Hölder's inequality, we get

$$
\begin{aligned}
\left|M^{(n)}\right|^{2 s} & \leq A_{1}^{2 s-1} \sum_{\chi_{1} \in \widehat{\mathbf{F}}_{q}^{\times}}\left|\sum_{\substack{\chi_{2} \in \mathcal{A}_{2} \\
\chi_{1} \chi_{2} \lambda \neq \mathbf{1}}} q^{-n} G\left(\chi_{2}\right)^{n}{\overline{G\left(\chi_{1} \chi_{2} \lambda\right.}}^{n}\right|^{2 s} \\
& =A_{1}^{2 s-1} \sum_{\boldsymbol{\eta}, \boldsymbol{\rho} \in \mathcal{A}_{2}^{s}} S(\boldsymbol{\eta}, \boldsymbol{\rho}) \prod_{i=1}^{s} q^{-n}{\overline{G\left(\eta_{i}\right)^{n}}}^{n} G\left(\rho_{i}\right)^{n},
\end{aligned}
$$

where

$$
S(\boldsymbol{\eta}, \boldsymbol{\rho})=\sum_{\chi \in \widehat{\mathbf{F}}_{q}^{\times} \backslash \mathcal{C}_{\boldsymbol{\eta}, \boldsymbol{\rho}}} \prod_{i=1}^{s} q^{-n} G\left(\chi \eta_{i} \lambda\right)^{n}{\overline{G\left(\chi \rho_{i} \lambda\right.}}^{n},
$$

Here $\mathcal{C}_{\boldsymbol{\eta}, \boldsymbol{\rho}}=\left\{\eta_{1}^{-1} \lambda^{-1}, \ldots, \eta_{s}^{-1} \lambda^{-1}, \rho_{1}^{-1} \lambda^{-1}, \ldots, \rho_{s}^{-1} \lambda^{-1}\right\}$. Each summand of $S(\boldsymbol{\eta}, \boldsymbol{\rho})$ has absolute value 1 , which implies the trivial bound $|S(\boldsymbol{\eta}, \boldsymbol{\rho})| \leq q-2$. If $\boldsymbol{\rho}$ is not a 
permutation of $\boldsymbol{\eta}$, then, removing the redundant factors in the product and applying Katz's bound [K, page 162 , line 8$]$ to the sum extended to $\widehat{\mathbf{F}_{q}^{\times}}$, we get

$$
|S(\boldsymbol{\eta}, \boldsymbol{\rho})| \leq\left|\sum_{\chi \in \widehat{\mathbf{F}_{q}^{\times}}}\right|+\left|\sum_{\chi \in \mathcal{C}_{\boldsymbol{\eta}, \boldsymbol{\rho}}}\right| \leq \operatorname{sn} \frac{q-1}{\sqrt{q}}+\frac{2 s}{\sqrt{q^{n}}}
$$

where we used the fact $\# \mathcal{C}_{\boldsymbol{\eta}, \boldsymbol{\rho}} \leq 2 s$. Therefore,

$$
\left|M^{(n)}\right|^{2 s} \leq A_{1}^{2 s-1}\left(s ! A_{2}^{s} q+A_{2}^{2 s} s(n \sqrt{q}+1)\right) .
$$

For the proof of (4), let

$$
\tilde{M}^{(n)}=\sum_{\substack{\chi_{1} \in \mathcal{A}_{1} \\ \chi_{2} \in \mathcal{A}_{2}}} q^{-\frac{(m+1) n}{2}} G\left(\chi_{1}\right)^{n} \cdots G\left(\chi_{m}\right)^{n}{\overline{G\left(\chi_{1} \chi_{2} \lambda\right)}}^{n} .
$$

Then

$$
\left|\tilde{M}^{(n)}-M^{(n)}\right| \leq \min \left\{A_{1}, A_{2}\right\} q^{-n / 2} \leq \sqrt{A_{1} A_{2}} q^{-n / 2}
$$

and

$$
\tilde{M}^{(n)}=q^{-\frac{(m+1) n}{2}} G\left(\chi_{3}\right)^{n} \ldots G\left(\chi_{m}\right)^{n} \sum_{\substack{\chi_{1} \in \mathcal{A}_{1} \\ \chi_{2} \in \mathcal{A}_{2}}} \sum_{a \in \mathbf{F}_{q}^{\times}} G\left(\chi_{1}\right)^{n} G\left(\chi_{2}\right)^{n} \overline{\chi_{1}}(a) \overline{\chi_{2}}(a) \bar{\lambda}(a) \overline{\operatorname{Kl}_{n}(a)} .
$$

Here we used

$$
G(\chi)^{n}=\sum_{a \in \mathbf{F}_{q}^{\times}} \mathrm{Kl}_{n}(a) \chi(a)
$$

where

$$
\mathrm{Kl}_{n}(a)=\sum_{\substack{a_{1}, \ldots, a_{n} \in \mathbf{F}_{q}^{\times} \\ a_{1} \cdots a_{n}=a}} \psi\left(a_{1}+\cdots+a_{n}\right)
$$

is the Kloosterman sum. Applying Deligne's bound [D, (7.1.3)]

$$
\left|\mathrm{Kl}_{n}(a)\right| \leq n q^{(n-1) / 2},
$$

we get

$$
\left|\tilde{M}^{(n)}\right| \leq n q^{-1 / 2} \sum_{a \in \mathbf{F}_{q}^{\times}}\left|g_{1}(a) g_{2}(a)\right|,
$$

where $g_{i}(a)=\sum_{\chi \in \mathcal{A}_{i}} q^{-n / 2} G(\chi)^{n} \bar{\chi}(a)$. Since

$$
\sum_{a \in \mathbf{F}_{q}^{\times}}\left|g_{i}(a)\right|^{2}=\sum_{a \in \mathbf{F}_{q}^{\times}} \sum_{\eta, \rho \in \mathcal{A}_{i}} q^{-n} \overline{G(\eta)}^{n} G(\rho)^{n} \eta \bar{\rho}(a)=A_{i}(q-1),
$$

we have

$$
\sum_{a \in \mathbf{F}_{q}^{\times}}\left|g_{1}(a) g_{2}(a)\right| \leq \sqrt{A_{1} A_{2}}(q-1)
$$

by Cauchy's inequality. Therefore,

$$
\left|M^{(n)}\right| \leq \sqrt{A_{1} A_{2}} q^{-1 / 2}(n(q-1)+1) .
$$


Proof of Theorem 1. Given nonnegative functions $f$ and $g$, we write $f \ll g$ for the existence of an absolute constant $C_{0}$ such that $f \leq C_{0} g$. Let $D=D\left(\mathcal{A}_{1} \times \mathcal{A}_{2} \times \mathcal{B}\right)$. We apply the Erdős-Turán inequality [ET, Theorem III]. For any real number $K \geq 1$, we have

$$
D \ll \frac{1}{K}+\sum_{1 \leq n \leq K} \frac{M^{(n)}}{n \# \mathcal{A}^{\circ}} .
$$

Note that $\# \mathcal{A}^{\circ} \geq\left(A_{1}-1\right) A_{2} B$.

For (11), taking $C \geq 2$, we may assume $A_{1} \geq(2 s)^{2 s+1} \sqrt{q}$ since $D \leq 1$. By (3), we get

$$
\begin{aligned}
D & \ll \frac{1}{K}+\sum_{1 \leq n \leq K}\left(\frac{\sqrt{s}}{n}\left(\frac{q}{A_{1} A_{2}^{s}}\right)^{\frac{1}{2 s}}+\frac{1}{n}\left(\frac{s n \sqrt{q}}{A_{1}}\right)^{\frac{1}{2 s}}\right) \\
& \ll \frac{1}{K}+\sqrt{s}\left(\frac{q}{A_{1} A_{2}^{s}}\right)^{\frac{1}{2 s}}(1+\log K)+s\left(\frac{s K \sqrt{q}}{A_{1}}\right)^{\frac{1}{2 s}} .
\end{aligned}
$$

Taking $K=s^{-1}\left(\frac{A_{1}}{\sqrt{q}}\right)^{\frac{1}{2 s+1}}$, the first and third terms of the right-hand side of (5) are both equal to $s\left(\frac{\sqrt{q}}{A_{1}}\right)^{\frac{1}{2 s+1}}$. For the remaining term, note that $K \geq 2$ by our assumption on $A_{1}$ and thus $1+\log K \ll \log K \ll s^{-1} \log q$. Here in the last estimate we used the fact $A_{1}<q$, which implies $K<q^{\frac{1}{4 s+2}}$. The bound (11) follows.

For (2), we may assume $A_{1} A_{2} \geq q$ and, by symmetry, $A_{1} \geq \sqrt{q}$. By (4), we get

$$
D \ll \frac{1}{K}+K \sqrt{\frac{q}{A_{1} A_{2}}} .
$$

We conclude by taking $K=\left(\frac{q}{A_{1} A_{2}}\right)^{-1 / 4}$.

Proof of Corollary 2. Part (ii) is obvious from (2). For (i), it suffices to show the existence of a constant $C^{\prime}>0$ (depending on $\epsilon$ and $c$ ) such that

$$
D\left(\mathcal{A}_{1} \times \mathcal{A}_{2} \times \mathcal{B}\right) \leq C^{\prime}\left((\log \log q)^{-1}+\sqrt{\frac{(\log q)^{\frac{1}{\epsilon}-1}(\log \log q)}{A_{2}}}\right)
$$

To see this, we take $s=\left\lceil\frac{\epsilon \log q}{2 \log \log q}\right\rceil$ in (1) and note that there exist constants $c^{\prime}$ and $c^{\prime \prime}$ (depending on $\epsilon$ and $c$ ) such that

$$
\begin{aligned}
\log \left(s\left(\frac{\sqrt{q}}{A_{1}}\right)^{\frac{1}{2 s+1}}\right) & \leq \log s+\frac{\log \left(c^{-1} q^{-\epsilon}\right)}{2 s+1} \\
& \leq(\log \log q-\log \log \log q)-\log \log q+c^{\prime} \\
& =-\log \log \log q+c^{\prime} \\
\log \left(\frac{1}{\sqrt{s}}\left(\frac{q}{A_{1}}\right)^{\frac{1}{2 s}} \log q\right) & \leq-\frac{1}{2} \log s+\frac{\log \left(c^{-1} q^{\frac{1}{2}-\epsilon}\right)}{2 s}+\log \log q \\
& \leq-\frac{1}{2}(\log \log q-\log \log \log q)+\left(\frac{1}{2 \epsilon}-1\right) \log \log q+\log \log q+c^{\prime \prime} \\
& =\left(\frac{1}{2 \epsilon}-\frac{1}{2}\right) \log \log q+\frac{1}{2} \log \log \log q+c^{\prime \prime} .
\end{aligned}
$$




\section{Acknowledgments}

We wish to thank Ping Xi for many helpful comments, especially his suggestion on the presentation of the proof of (4). We are grateful to Nick Katz and Zhiyong Zheng for encouragement. We thank the referee for useful comments. We acknowledge the support of Princeton University, where part of this work was done during a visit.

\section{References}

[dlBSSV] R. de la Bretèche, M. Sha, I. E. Shparlinski, and J. F. Voloch, The Sato-Tate distribution in thin parametric families of elliptic curves, Math. Z. 290 (2018), no. 3-4, 831-855, DOI 10.1007/s00209-018-2042-0. MR3856834 㤷

[D] P. Deligne, Application de la formule des traces aux sommes trigonométriques, Cohomologie étale, Lecture Notes in Mathematics, vol. 569, Springer-Verlag, Berlin, 1977.

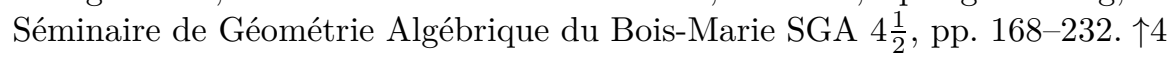

[ET] P. Erdös and P. Turán, On a problem in the theory of uniform distribution. I, II, Nederl. Akad. Wetensch., Proc. 51 (1948), 1146-1154, 1262-1269 = Indagationes Math. 10,

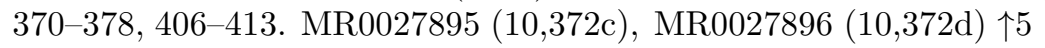

[K] N. M. Katz, Gauss sums, Kloosterman sums, and monodromy groups, Annals of Mathematics Studies, vol. 116, Princeton University Press, Princeton, NJ, 1988. MR955052 (91a:11028) $\uparrow 2$, 4

[KZ] N. M. Katz and Z. Zheng, On the uniform distribution of Gauss sums and Jacobi sums, Analytic number theory, Vol. 2 (Allerton Park, IL, 1995), Progr. Math., vol. 139,

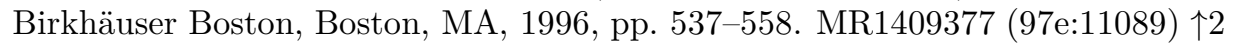

[LZZ] Q. Lu, W. Zheng, and Z. Zheng, On the distribution of Jacobi sums, J. Reine Angew. Math. 741 (2018), 67-86, DOI 10.1515/crelle-2015-0087. MR3836143 悓

[S] I. E. Shparlinski, On the distribution of arguments of Gauss sums, Kodai Math. J. 32 (2009), no. 1, 172-177, DOI 10.2996/kmj/1238594554. MR2518562 (2010b:11104) 败

[X] P. Xi, Equidistributions of Jacobi sums. arXiv:1809.04286v1, preprint. 怆 